\title{
4. SNAPSHOT OF CHILD HEALTH, NSW, 2001
}

\section{SNAPSHOT OF CHILD HEALTH, NSW, 2001}

\begin{tabular}{|c|c|c|c|}
\hline Topic & Issues & Indicator & Result (\%) \\
\hline \multicolumn{4}{|c|}{ Determinants of health } \\
\hline & Social support & $\begin{array}{l}\text { Parents or carers who reported having friends who make them feel } \\
\text { safe, secure and happy }\end{array}$ & 95.7 \\
\hline & Social capital & $\begin{array}{l}\text { Parents or carers who reported being able to get help from a } \\
\text { neighbour to care for a child }\end{array}$ & 68.3 \\
\hline & & Parents or carers who reported most people can be trusted & 61.6 \\
\hline & & $\begin{array}{l}\text { Parents or carers who reported they had helped out at any local } \\
\text { group or organisation }\end{array}$ & 40.4 \\
\hline & $\begin{array}{l}\text { Early childhood } \\
\text { programs }\end{array}$ & $\begin{array}{l}\text { Children aged } 4 \text { years reported to have been currently attending } \\
\text { preschool or other regular early childhood programs }\end{array}$ & 92.8 \\
\hline \multicolumn{4}{|c|}{ Health behaviours } \\
\hline & Smoking in pregnancy & Mothers who reported smoking in pregnancy & 14.2 \\
\hline & Smoking in the home & $\begin{array}{l}\text { Parents or carers who reported that no-one living in their } \\
\text { household smoked }\end{array}$ & 65.7 \\
\hline & Infant sleeping position & Parents or carers who reported placing baby on its back to sleep & 62.4 \\
\hline & Folate in pregnancy & $\begin{array}{l}\text { Mothers who reported awareness of reasons for taking folate in } \\
\text { pregnancy }\end{array}$ & 61.6 \\
\hline & Breastfeeding & Mothers who reported their infant had ever been breastfed & 89.8 \\
\hline & Food intake & $\begin{array}{l}\text { Children reported to consume the recommended quantity of fruits } \\
\text { (includes fruit juice) }\end{array}$ & 92.7 \\
\hline & & $\begin{array}{l}\text { Children reported to consume the recommended quantities of } \\
\text { vegetables }\end{array}$ & 12.9 \\
\hline & Sun exposure & $\begin{array}{l}\text { Children reported to have always used sunscreen when out in the } \\
\text { sun for fifteen minutes or more in the previous summer }\end{array}$ & 86.6 \\
\hline & & $\begin{array}{l}\text { Children reported to wear protective clothing when out in the sun } \\
\text { for fifteen minutes or more in the previous summer }\end{array}$ & 82.4 \\
\hline & Physical activity & Children reported to participate in sports or physical activities & 91.9 \\
\hline & Physical inactivity & $\begin{array}{l}\text { Children reported to watch an average of two or more hours } \\
\text { television per day }\end{array}$ & 39.5 \\
\hline \multicolumn{4}{|c|}{ Health status } \\
\hline & Asthma & Children reported to have current asthma & 15.7 \\
\hline & & $\begin{array}{l}\text { Children with current asthma who had a written asthma } \\
\text { management plan }\end{array}$ & 43.6 \\
\hline & Oral health & $\begin{array}{l}\text { Children reported to have visited a dental professional in last } 12 \\
\text { months (excluding SOKS assessment) }\end{array}$ & 43.1 \\
\hline & $\begin{array}{l}\text { Emotional or } \\
\text { behavioural problems } \\
\text { Infant feeding }\end{array}$ & $\begin{array}{l}\text { Children reported to have any emotional or behavioural problems } \\
\text { in the last six months }\end{array}$ & 31.4 \\
\hline & problems & Infants reported to have feeding problems & 28.0 \\
\hline & $\begin{array}{l}\text { Infant behavioural } \\
\text { problems }\end{array}$ & Infants reported to have behavioural problems & 35.1 \\
\hline & $\begin{array}{l}\text { Toddler eating or } \\
\text { feeding problems }\end{array}$ & Toddlers reported to have eating or feeding problems & 7.5 \\
\hline & $\begin{array}{l}\text { Toddler physical } \\
\text { developmental } \\
\text { problems }\end{array}$ & Toddlers reported to have physical developmental problems & 3.2 \\
\hline \multicolumn{4}{|c|}{ Health services } \\
\hline & $\begin{array}{l}\text { Personal health } \\
\text { record }\end{array}$ & Children reported to have a personal health record & 94.0 \\
\hline & $\begin{array}{l}\text { Attendance at child } \\
\text { and family health }\end{array}$ & $\begin{array}{l}\text { Parents or carers who reported ever attending child and family } \\
\text { health services }\end{array}$ & 92.1 \\
\hline & services & $\begin{array}{l}\text { Parents or carers who reported currently attending child and family } \\
\text { health services }\end{array}$ & 28.9 \\
\hline & $\begin{array}{l}\text { Parental support } \\
\text { services }\end{array}$ & $\begin{array}{l}\text { Parents or carers who reported they needed parental support } \\
\text { services }\end{array}$ & 68.6 \\
\hline & & $\begin{array}{l}\text { Parents or carers who reported they needed parental support } \\
\text { services but did not access them }\end{array}$ & 19.0 \\
\hline & Home visiting & Parents or carers who reported they had received a home visit & 36.3 \\
\hline
\end{tabular}

